\title{
Primal-dual stability in continuous linear optimization
}

Received: date / Accepted: date

\begin{abstract}
Any linear (ordinary or semi-infinite) optimization problem, and also its dual problem, can be classified as either inconsistent or bounded or unbounded, giving rise to nine duality states, three of them being precluded by the weak duality theorem. The remaining six duality states are possible in linear semi-infinite programming whereas two of them are precluded in linear programming as a consequence of the existence theorem and the nonhomogeneous Farkas Lemma. This paper characterizes the linear programs and the continuous linear semi-infinite programs whose duality state is preserved by sufficiently small perturbations of all the data. Moreover, it shows that almost all linear programs satisfy this stability property.
\end{abstract}

Keywords linear programming · linear semi-infinite programming · stability

\section{Introduction}

Any finite linear programming (LP) problem with $n \geq 2$ decision variables can be formulated in canonical form as

$$
\begin{aligned}
& P: \text { Min } c^{\prime} x \\
& \quad \text { s.t. } \quad a_{t}^{\prime} x \geq b_{t}, \quad t \in T,
\end{aligned}
$$

Miguel A. Goberna

Dep. of Statistics and Oper. Res.,University of Alicante, 03080 Alicante, Spain. E-mail: mgoberna@ua.es Research supported by DGES and FEDER, Grant MTM2005-08572-C03-01

Maxim I. Todorov

Dep. of Physics and Mathematics, UDLA, 72820 San Andrés Cholula, Puebla, Mexico. On leave from IMI-BAS, Sofia, Bulgaria.

E-mail: (maxim.todorov@udlap.mx)

Research partially supported by CONACyT of MX.Grant 44003 
where $T$ is a finite index set, $c$ and $x$ are vectors in $\mathbb{R}^{n}$, and $a_{t}$ and $b_{t}$ are the images of $t \in T$ by mean of the mappings $a: T \longmapsto \mathbb{R}^{n}$ and $b: T \longmapsto \mathbb{R}$, respectively. The dual problem of $P$ is the following $L P$ problem in standard form:

$$
\begin{aligned}
D: & \operatorname{Max} \\
\text { s.t. } & \sum_{t \in T} \lambda_{t} b_{t} \\
& \sum_{t \varepsilon T} \lambda_{t} a_{t}=c, \\
& \lambda_{t} \geq 0, t \in T .
\end{aligned}
$$

Obviously, $P$ and $D$ involve the same data, so that both problems can be represented by the triple $\pi:=(a, b, c)$.

In LP we are familiar with the four mutually exclusive and collectively exhaustive duality states that can occur when $P$ and $D$ are classified as either inconsistent or bounded (i.e., with finite optimal value) or unbounded. The classification of $\pi$ into its corresponding duality state is interesting for different reasons. For instance, solving $P$ and $D$ simultaneously can be reduced to solving the associated primal-dual system,

$$
\left\{a_{t}^{\prime} x \geq b_{t}, t \in T ; \sum_{t \varepsilon T} \lambda_{t} a_{t}=c ; \lambda_{t} \geq 0, t \in T ; c^{\prime} x=\sum_{t \in T} \lambda_{t} b_{t}\right\},
$$

if and only if both problems are bounded (this is the class of LP problems which can be solved by means of numerical methods for linear inequality systems). Moreover, $P$ and $D$ have different optimal values if and only if they are inconsistent. The first systematic study of the duality states in mathematical programming appeared in [5] (paper revisited in [23]), where the authors considered versions for conic programming, convex programming and a particular class of linear semi-infinite programming (LSIP) problems. This work was extended to reflexive spaces in [21]. Duality states in semidefinite programming were extensively analyzed with computational issues in [35] and compared with the LSIP counterpart in [25].

If $T$ is an infinite compact Hausdorff topological space and the functions $a$ and $b$ are continuous on $T$, then the LSIP problem $P$ is called continuous. The so-called Haar's dual problem of $P$ is the extension of $D$ consisting of taking as space of variables the linear space of all the functions $\lambda: T \mapsto \mathbb{R}$ such that $\lambda_{t}=0$ for all $t \in T$ except maybe for a finite number of indices. This space is denoted by $\mathbb{R}^{(T)}$ (the space of generalized finite sequences) and its positive cone (formed by the non-negative generalized finite sequences) by $\mathbb{R}_{+}^{(T)}$. Thus, the dual problem of $P$ reads

$$
\begin{aligned}
D: & \operatorname{Max} \\
\text { s.t. } & \sum_{t \in T} \lambda_{t} b_{t} \lambda_{t} a_{t}=c, \lambda \in \mathbb{R}_{+}^{(T)} .
\end{aligned}
$$

If $|T|<\infty$ and we consider $T$ equipped with the discrete topology, then $T$ is compact Hausdorff and the coefficient functions are trivially continuous. Moreover, $\mathbb{R}^{(T)}=\mathbb{R}^{T}$, so that the problems in (1) and (2) coincide. For this reason, we say that both LP and continuous LSIP problems are continuous 
linear optimization problems (assuming implicitly that $T$ is equipped with the discrete topology when $|T|<\infty)$.

The continuity property of $P$ ensures nice theoretical properties (e.g., in the duality context), although the boundedness of one of the problems does not entail their solvability and the coincidence of optimal values, and has computational implications (e.g., continuity guarantees the convergence of LSIP discretization algorithms). Among the recent applications of continuous LSIP let us mention that $P$ arises in functional approximation ([12], $[13])$, separation $([22])$, finance $([24])$, Bayesian statistics ([29]) and the design of telecommunications networks ([11], [28], [34]), whereas $D$ has been used in robust Bayesian analysis ([6]) and optimization under uncertainty ([1]); another type of dual problem for $P$ closely related to $D$, whose space of variables is formed by regular Borel measures, has been used in optimal control ([32], [33]).

We denote by $v^{P}(\pi)\left(v^{D}(\pi)\right)$ the optimal value of $P(D)$, defining as usual $v^{P}(\pi)=+\infty\left(v^{D}(\pi)=-\infty\right.$, respectively) when the corresponding problem is inconsistent. Since $P$ and $D$ can be either inconsistent (IC) or bounded (B) or unbounded (UB), crossing both criteria we get at most nine possible duality states, which are reduced to six by the weak duality theorem: $v^{D}(\pi) \leq v^{P}(\pi)$. The possible duality states in continuous linear optimization are enumerated in Diagram 1 (according to the duality theorem, the duality states 5 and 6 are impossible in LP):

\begin{tabular}{|c||c|c|c|}
\hline$D \backslash P$ & IC & B & UB \\
\hline \hline IC & 4 & 5 & 2 \\
\hline B & 6 & 1 & \\
\hline UB & 3 & & \\
\hline
\end{tabular}

\section{Diagram 1}

We associate with the given nominal triple $\pi=(a, b, c)$ the set of perturbed triples which are admissible in the sense that they preserve the numbers of constraints and variables in $P$ and $D$ as well as the continuity of $P$. This set, called space of parameters, is

$$
\Pi:=\mathbb{R}^{n|T|+|T|+n},
$$

if $|T|<\infty$, and

$$
\Pi:=\mathcal{C}(T)^{n} \times \mathcal{C}(T) \times \mathbb{R}^{n},
$$

otherwise. Observe that $\Pi$ only depends on $n$ and $T$. The perturbations generating $\Pi$ could be the consequence of rounding errors or measurement errors.

This is the first paper analyzing the effect on the duality state of perturbing the data. More in detail, our main objective is characterizing those parameters $\pi=(a, b, c)$ which are primal-dual stable (relative to the duality states considered in this paper) in the sense that sufficiently small admissible perturbations of $a, b$, and $c$ preserve its duality state. In order to reformulate this objective in topological terms, we denote by $\Pi_{i}$ the set of parameters in the duality state $i, i=1, \ldots, 6$ (e.g., $\pi \in \Pi_{6}$ when $P$ is inconsistent and 
$D$ is finite valued). Recall that, in LP, $\Pi_{5}=\Pi_{6}=\emptyset$. The null element of $\Pi$ obviously belongs to $\Pi_{1}$ and the non-empty sets of $\left\{\Pi_{i}, i=1, \ldots, 6\right\}$ are (non-convex) cones providing a partition of the space of parameters $\Pi$. Then, defining a suitable topology on $\Pi$, we characterize the interior of $\Pi_{i}$, $i=1, \ldots, 6$, in terms of relationships between points and sets in finite dimensional Euclidean spaces. In [36, Proposition 1.5] it is shown that the interior of $\Pi_{1}$ is the class of parameters such that sufficiently small perturbations provide primal solvable problems. Secondary objectives of the paper are the characterization of those duality states for which any of its members can be approached by means of stable parameters (i.e., those $\Pi_{i}$ such that $\Pi_{i}$ is contained in the closure of its interior) and to prove that most perturbations of the nominal parameter are primal-dual stable.

As most of the works on perturbation theory in continuous LSIP (e.g., the classical paper [30]), we measure the size of a perturbation by means of the metric derived from the norm of the uniform convergence, i.e., given $\pi^{i}=\left(a^{i}, b^{i}, c^{i}\right) \in \Pi, i=1,2$, we define

$$
d\left(\pi^{1}, \pi^{2}\right)=\max \left\{\left\|c^{1}-c^{2}\right\|_{\infty}, \max _{t \in T}\left\|\left(\begin{array}{c}
a_{t}^{1} \\
b_{t}^{1}
\end{array}\right)-\left(\begin{array}{c}
a_{t}^{2} \\
b_{t}^{2}
\end{array}\right)\right\|_{\infty}\right\} .
$$

If $T$ is a compact Hausdorff space, then the Banach space $\Pi$ can be seen as a topological subspace of the space of general LSIP problems equipped with the pseudometric of the uniform convergence (defined, as in [20], by replacing "max" with "sup" in (3)) or even as a subspace of the space of convex semiinfinite programming (CSIP) problems equipped with the pseudometric of the uniform convergence on compact sets defined in [15] (the last two spaces of parameters are well-defined even when $T$ is not a topological space). The semicontinuity properties of the primal feasible set, the primal optimal set and the primal optimal value function $v^{P}$ have been characterized during the 80's for continuous LSIP ([7], [14], etc.), during the 90's for general LSIP ([17], [18], [16], etc.) and during the present decade for CSIP ([26], [15]). Observe that a sufficient condition for the semicontinuity (in certain sesnse) of one of the mentioned mappings at a given triple of a certain space of parameters is also a sufficient condition for the semicontinuity of the restriction of this mappings to any topological subspace containing that triple, whereas the situation is the opposite regarding the necessary conditions, i.e., the stability theory for certain class of problems is not subsumed by the corresponding theory in a more general framework. Recent works on stability in LSIP deal with quantitative aspects as the distance to ill-posedness and error bounds in general LSIP ([9], [10]) and metric regularity in continuous LSIP ([8]), where the Banach property of the space of right-hand side functions plays a crucial role.

As a general rule, it is difficult to get dual counterparts for the results mentioned in the previous paragraph (due to the poor topological properties of the spaces of dual variables and right-hand side functions in general LSIP), whereas the situation is more favorable in continuous LSIP. For this reason, the semicontinuity of the dual feasible set in general LSIP was only characterized in [19], whereas the characterizations of the semicontinuity of $v^{D}$ and the dual optimal set are still open problems. Concerning the interior 
of the sets of the dual partition (corresponding to the entries of the three rows in Diagram 1), which are basic tools in this paper (see Lemma 2), the interior of the class of triples providing a stable inconsistent dual problem in continuous LSIP was characterized quite late ([19]), whereas no characterization has been obtained up to now for either general LSIP or CSIP (where the Haar's dual problem must be replaced by another one, e.g., the Lagrangian dual of $P$ ).

Let us observe that our approach to primal-dual stability in continuous linear optimization is not intrinsic in the sense that it depends on the topology defined on $\Pi$. For instance, since each triple $\pi=(a, b, c)$ could be identified with a couple $(C, c) \in 2^{\mathbb{R}^{n+1}} \times \mathbb{R}^{n}$, where $C$ is a certain closed set (e.g., either the compact set $\left\{\left(a_{t}, b_{t}\right), t \in T\right\}$ or the closure of the characteristic cone of $\pi$ defined in Section 2), it is possible to consider $\Pi$ equipped with the Hausdorff topology, the bounded Hausdorff topology ([3], [2], [31]), or any other topology on the space of closed sets ([4]). We prefer to use the topology of the uniform convergence in the parameter space $\Pi$ first, because this topology makes sense in practice (so that it has been extensively analyzed) and second, because the representation of $\pi$ in $2^{\mathbb{R}^{n+1}} \times \mathbb{R}^{n}$ affects the dual problem, i.e., this approach is only suitable for the stability analysis of the primal problem (in particular, the stability of the primal feasible set has been analyzed in [27] taking as $C$ the intersection of the closure of the characteristic cone of $\pi$ with the closed unit ball, obtaining results which are not valid for the topology of the uniform convergence). Similar difficulties appear if we consider, f.i., bounded Hausdorff topology in the image space of the different mappings arising from the continuous linear optimization.

The paper is organized as follows: Section 2 contains additional notation and some basic results to be used later, Section 3 analyzes primal-dual stability in LP and Section 4 provides similar results in continuous LSIP. We have split the study of the primal-dual stability depending on the cardinality of $T$ due to the outstanding advantages of the finite case in comparison with the infinite one: first, the duality state of a parameter is determined by its primal and dual feasibility and, second, any perturbation is admissible (preserving continuity under perturbations, in LSIP, requires the use of rather sophisticated tools).

\section{Preliminaries}

Let us introduce the necessary notation. $0_{p}$ denotes the null-vector in $\mathbb{R}^{p}$, the $j$ th element of the canonical basis of $\mathbb{R}^{p}$ is $e_{j}$ whereas $e=e_{1}+\ldots+e_{p}$. The Euclidean and the $l_{\infty}$ (or Chebyshev) norms (in any of the spaces $\mathbb{R}^{p}$ and $\mathcal{C}(T)$ ) are represented by $\|\cdot\|$ and $\|\cdot\|_{\infty}$, respectively. Given a non-empy set $X \subset \mathbb{R}^{p}$, conv $X$ and cone $X:=\mathbb{R}_{+}$conv $X$ denote the convex hull and the conical convex hull of $X$, respectively (it is also assumed that cone $\emptyset=\left\{0_{p}\right\}$ ). If $X$ is convex, $\operatorname{dim} X$ denotes its dimension. From the topological side, if $X$ is a subset of any topological space, int $X, \operatorname{cl} X$ and $\mathrm{bd} X$ represent the interior, the closure and the boundary of $X$, respectively. Finally, $\lim _{r}$ should be interpreted as $\lim _{r \rightarrow \infty}$. 
The next result on Chebyshev functional approximation is used frequently throughout the paper. The proof of part (iii) is an easy exercise when $|T|<\infty$ (otherwise we need Urisohn's lemma).

Lemma 1 Let $T$ be a compact Hausdorff space, $\bar{x} \in \mathbb{R}^{n}$ and $x \in \mathcal{C}(T)^{n}$. Then the following statements hold:

(i) If $\bar{x} \in$ int cone $\{x(t), t \in T\}$, then there exists $\varepsilon>0$ such that $\bar{y} \in$ int cone $\{y(t), t \in T\}$ for all $\bar{y} \in \mathbb{R}^{n}$ and $y \in \mathcal{C}(T)^{n}$ such that $\|\bar{y}-\bar{x}\|_{\infty}<\varepsilon$ and $\|y-x\|_{\infty}<\varepsilon$.

(ii) If $0_{n} \neq \bar{x} \notin$ int cone $\{x(t), t \in T\}$, then for all $\varepsilon>0$ there exists $y \in \mathcal{C}(T)^{n}$ such that $\bar{x} \notin$ cone $\{y(t), t \in T\}$ and $\|y-x\|_{\infty}<\varepsilon$.

(iii) If $0_{n} \neq \bar{x} \in$ cone $\{x(t), t \in T\}$ and $|T| \geq n$, then for all $\varepsilon>0$ there exists $y \in \mathcal{C}(T)^{n}$ such that $\bar{x} \in$ int cone $\{y(t), t \in T\}$ and $\|y-x\|_{\infty}<\varepsilon$.

(iv) If $\bar{x} \in \operatorname{conv}\{x(t), t \in T\}$ and $|T| \geq n+1$, then for all $\varepsilon>0$ there exists $y \in \mathcal{C}(T)^{n}$ such that $\bar{x} \in$ int cone $\{y(t), t \in T\}$ and $\|y-x\|_{\infty}<\varepsilon$.

Proof (i) It is a straightforward consequence of [17, Lemma 4.2].

(ii) By the separation theorem and the supporting hyperplane theorem, applied to $\bar{x}$ and cl cone $\{x(t), t \in T\}$, there exists $d \in \mathbb{R}^{n} \backslash\left\{0_{n}\right\}$ such that $d^{\prime} \bar{x} \leq 0$ and $d^{\prime} x(t) \geq 0$ for all $t \in T$.

Let $y:=x+\frac{\varepsilon d}{2\|d\|_{\infty}} \in \mathcal{C}(T)^{n}$. Obviously, $\|y-x\|_{\infty}<\varepsilon$. Assume that $\bar{x} \in$ cone $\{y(t), t \in T\}$. Then we can write $\bar{x}=\sum_{t \in T} \lambda_{t} y(t), \lambda \in \mathbb{R}_{+}^{(T)}$. Since we assume $\bar{x} \neq 0_{n}, \sum_{t \in T} \lambda_{t}>0$ and so,

$$
d^{\prime} \bar{x}=\sum_{t \in T} \lambda_{t} d^{\prime} y(t)=\sum_{t \in T} \lambda_{t} d^{\prime}\left(x(t)+\frac{\varepsilon d}{2\|d\|_{\infty}}\right) \geq\left(\sum_{t \in T} \lambda_{t}\right) \frac{\varepsilon\|d\|^{2}}{2\|d\|_{\infty}}>0
$$

in contradiction with $d^{\prime} \bar{x} \leq 0$.

(iii) First we prove the existence of $z \in \mathcal{C}(T)^{n}$ such that $\|z-x\|_{\infty}<\frac{\varepsilon}{2}$, $\operatorname{dim} \operatorname{span}\{z(t), t \in T\}=n$ and $\bar{x} \in \operatorname{cone}\{z(t), t \in T\}$.

By Carathéodory's theorem for cones, we can write $\bar{x}=\sum_{i=1}^{m} \lambda_{i} x\left(t_{i}\right), t_{i} \in T$ and $\lambda_{i} \geq 0, i=1, \ldots, m$, and $\left\{x\left(t_{i}\right), i=1, \ldots, m\right\}$ linearly independent. If $m=$ $n$ we can choose $z:=x$. Otherwise take $n-m$ elements $t_{m+1}, \ldots, t_{n}$ arbitrarily in $T \backslash\left\{t_{1}, \ldots, t_{m}\right\}$ (we are assuming that $|T| \geq n$ ). We define $u_{i}:=0_{n}, i=$ $1, \ldots, m$. If $x\left(t_{m+1}\right) \notin \operatorname{span}\left\{x\left(t_{i}\right), i=1, \ldots, m\right\}$, we define $u_{m+1}:=0_{n}$. Otherwise, we choose $u_{m+1} \in \mathbb{R}^{n}$ such that $\left\{x\left(t_{i}\right), i=1, \ldots, m ; x\left(t_{m+1}\right)+u_{m+1}\right\}$ is linearly independent and $\left\|u_{m+1}\right\|_{\infty}<\frac{\varepsilon}{2 n}$. By induction, we can select $u_{i} \in \mathbb{R}^{n}, i=m+1, \ldots, n$, such that $\left\{x\left(t_{i}\right)+u_{i}, i=1, \ldots, n\right\}$ is a basis of $\mathbb{R}^{n}$ and $\left\|u_{i}\right\|_{\infty}<\frac{\varepsilon}{2 n}, i=1, \ldots, n$. By Urisohn's lemma there exist continuous functions $p_{i}: T \stackrel{0}{\rightarrow}[0,1], i=1, \ldots, n$, such that

$$
p_{i}\left(t_{j}\right)=\left\{\begin{array}{l}
1, \text { if } j=i \\
0, \text { otherwise }
\end{array}\right.
$$


for each $i, j=1, \ldots, n$. It is easy to see that $z(t):=x(t)+\sum_{i=1}^{n} p_{i}(t) u_{i}$ satisfies all the requirements. If $\bar{x} \in \operatorname{int}$ cone $\{z(t), t \in T\}$, we have finished. So we assume that $\bar{x} \in \mathrm{bd}$ cone $\{z(t), t \in T\}$.

Now we perturb the function $z$ in order to get another function $y \in \mathcal{C}(T)^{n}$ such that $\|y-x\|_{\infty}<\varepsilon$ and $\bar{x} \in \operatorname{int}$ cone $\{y(t), t \in T\}$.

Since dim cone $\{z(t), t \in T\}=n$ and $\bar{x} \in$ bd cone $\{z(t), t \in T\}$, by the accessibility lemma, there exists a sequence $\left\{v^{r}\right\}_{r=1}^{\infty} \subset$ int cone $\{z(t), t \in T\}$ such that $\lim _{r} v^{r}=\bar{x} \neq 0_{n}$. Obviously, $\bar{x} \neq v^{r}$ for all $r$ and we can assume also that $v^{r} \neq 0_{n}$ for all $r$. Given $r \in \mathbb{N}$, let $g_{r}$ be an orthogonal transformation in $\mathbb{R}^{n}$ such that

$$
g_{r}\left(\frac{\bar{x}}{\|\bar{x}\|}\right)=\frac{v^{r}}{\left\|v^{r}\right\|}
$$

and

$$
\left\|g_{r}(u)-u\right\| \leq\left\|\frac{v^{r}}{\left\|v^{r}\right\|}-\frac{\bar{x}}{\|\bar{x}\|}\right\|
$$

for all $u \in \mathbb{R}^{n}$ such that $\|u\|=1$ (a natural choice for $g_{r}$ is the rotation in the plane span $\left\{\bar{x}, v^{r}\right\}$ such that (4) holds, and the identity on the orthogonal subspace). Since

we have

$$
g_{r}(\bar{x})=\frac{\|\bar{x}\|}{\left\|v^{r}\right\|} v^{r} \in \text { int cone }\{z(t), t \in T\}
$$

$$
\bar{x} \in \operatorname{int} \text { cone }\left\{g_{r}^{-1}[z(t)], t \in T\right\}, r=1,2, \ldots
$$

Let $I_{n}$ be the identity mapping in $\mathbb{R}^{n}$ and let $k:=\max \{\|z(t)\|, t \in T\}$. Given $t \in T$, (5) yields

$$
\begin{gathered}
\left\|g_{r}^{-1}[z(t)]-z(t)\right\|_{\infty} \leq\left\|g_{r}^{-1}[z(t)]-z(t)\right\|=\left\|z(t)-g_{r}[z(t)]\right\| \\
\leq\left\|I_{n}-g_{r}\right\|\|z(t)\| \leq k\left\|\frac{v^{r}}{\left\|v^{r}\right\|}-\frac{\bar{x}}{\|\bar{x}\|}\right\| .
\end{gathered}
$$

Since $\lim _{r}\left\|\frac{v^{r}}{\left\|v^{r}\right\|}-\frac{\bar{x}}{\|\bar{x}\|}\right\|=0$, there exists $r_{0} \in \mathbb{N}$ such that

$$
\left\|g_{r_{0}}^{-1}[z(t)]-z(t)\right\|_{\infty}<\frac{\varepsilon}{2} \text { for all } t \in T .
$$

Finally, it is easy to see that the composite function $y:=g_{r_{0}}^{-1} \circ z$ satisfies all the requirements.

(iv) Assume $\bar{x} \in \operatorname{conv}\{x(t), t \in T\}$ and $|T| \geq n+1$. Let $\varepsilon>0$. Since

$$
\left(\begin{array}{c}
\bar{x} \\
1
\end{array}\right) \in \operatorname{cone}\left\{\left(\begin{array}{c}
x(t) \\
1
\end{array}\right), t \in T\right\}
$$

by statement (iii) there exists $(y, f) \in \mathcal{C}(T)^{n+1}$ such that $\|(y, f)-(x, 1)\|_{\infty}<$ $\varepsilon$ and

$$
\left(\begin{array}{c}
\bar{x} \\
1
\end{array}\right) \in \operatorname{int} \text { cone }\left\{\left(\begin{array}{l}
y(t) \\
f(t)
\end{array}\right), t \in T\right\},
$$

so that $\bar{x} \in \operatorname{int}$ cone $\{y(t), t \in T\}$ and $\|y-x\|_{\infty}<\varepsilon$. 
Next we recall some basic results (most of them valid for general LSIP) we need on continuous linear optimization (all the proofs can be found in [16]). We associate with $\pi=(a, b, c)$ the feasible (optimal) sets of $D$ and $P$, which are denoted by $\Lambda$ and $F$ ( $\Lambda^{*}$ and $F^{*}$, respectively), the first and second moment cones of $\pi, M:=$ cone $\left\{a_{t}, t \in T\right\}$ and $N:=$ cone $\left\{\left(a_{t}, b_{t}\right), t \in T\right\}$, and the characteristic cone, $K:=N+\mathbb{R}_{+}\left\{\left(0_{n},-1\right)\right\}$. If $\pi$ satisfies the Slater condition, i.e., there exists $\bar{x} \in \mathbb{R}^{n}$ such that $a_{t}^{\prime} \bar{x}>b_{t}$ for all $t \in T$, then $N$ is closed. Moreover, if $P$ is consistent and $N$ is closed, then $K$ is closed too. If $D$ is consistent and $K$ is closed, then $D$ is solvable. The existence theorem establishes that $P$ is consistent if and only if $\left(0_{n}, 1\right) \notin \mathrm{cl} N$. In such a case, the non-homogeneous Farkas lemma establishes that the inequality $c^{\prime} x \geq d$ holds for all $x \in F$ if and only if $(c, d) \in \operatorname{cl} K$.

From now on, the perturbations of the nominal triple $\pi$ will be distinguished by means of upperscripts, and the same (either as subscripts or as superscripts) applies for their corresponding objects: $\pi^{r}=\left(a^{r}, b^{r}, c^{r}\right)$, $D_{r}, P_{r}, \Lambda_{r}, F_{r}$, and so on. We denote by

$$
\Pi_{c}^{P}=\left\{\pi^{1} \in \Pi \mid F_{1} \neq \emptyset\right\} \text { and } \Pi_{c}^{D}=\left\{\pi^{1} \in \Pi \mid \Lambda_{1} \neq \emptyset\right\}
$$

the classes of parameters providing primal and dual consistent problems. The last result in this section characterizes the primal and the dual stability of $\pi$ relative to the dichotomy consistent-inconsistent. Obviously, $\pi \in \Pi_{c}^{P}$ $\left(\pi \in \Pi_{c}^{D}\right)$ if and only if $\left(0_{n}, 1\right) \notin \mathrm{cl} N(c \in M$, respectively).

Lemma 2 The following statements are true:

(i) $\pi \in \operatorname{int} \Pi_{c}^{P}$ if and only if $P$ satisfies the Slater condition if and only if $0_{n+1} \notin \operatorname{conv}\left\{\left(a_{t}, b_{t}\right), t \in T\right\}$.

(ii) $\pi \in \operatorname{int}\left(\Pi \backslash \Pi_{c}^{P}\right)$ if and only if $\left(0_{n}, 1\right) \in \operatorname{int} N$.

(iii) $\pi \in \operatorname{int} \Pi_{c}^{D}$ if and only if $c \in \operatorname{int} M$.

(iv) $\pi \in \operatorname{int}\left(\Pi \backslash \Pi_{c}^{D}\right)$ if and only if there exists $y \in \mathbb{R}^{n}$ such that $c^{\prime} y<0$ and $a_{t}^{\prime} y>0$ for all $t \in T$.

Proof (i) It is [17, Theorem 3.1] and [37, Theorem 3.2].

(ii) It follows from $[17$, Theorems 6.3 and 6.4$]$.

(iii) It follows from $[19$, Theorem 5$]$.

(iv) It is $[19$, Theorem 10].

Other characterizations of $\pi \in \operatorname{int} \Pi_{c}^{P}$ and $\pi \in \operatorname{int}\left(\Pi \backslash \Pi_{c}^{P}\right)$ can be found in [9], where explicit formulae for the distance from $\pi$ to the corresponding boundary are also given.

\section{Primal-dual stability in linear programming}

Let $\Pi$ be the space of parameters corresponding to a given triple $\pi:=(a, b, c)$ such that $|T|<\infty$ and $n \geq 2$. Under these assumptions $\Pi_{5}=\Pi_{6}=\emptyset$ and the associated moment cones, $M$ and $N$, are polyhedral (and so closed), so 
that the four possible duality states for $\pi$ are characterized in Diagram 2 by means of the associated moment cones.

\begin{tabular}{|c|c|c|}
\cline { 2 - 3 } \multicolumn{1}{c|}{} & $\left(0_{n}, 1\right) \in N$ & $\left(0_{n}, 1\right) \notin N$ \\
\hline \hline$c \notin M$ & $\Pi_{4}$ & $\Pi_{2}$ \\
\hline$c \in M$ & $\Pi_{3}$ & $\Pi_{1}$ \\
\hline
\end{tabular}

Diagram 2

Theorem 1 The following statements are true:

(i) $\pi \in \operatorname{int} \Pi_{1}$ if and only if Slater condition holds and $c \in \operatorname{int} M$. Moreover, int $\Pi_{1}$ is dense in $\Pi_{1}$ if and only if $|T| \geq n$.

(ii) $\pi \in \operatorname{int} \Pi_{2}$ if and only if there exists $y \in \mathbb{R}^{n}$ such that $c^{\prime} y<0$ and $a_{t}^{\prime} y>0$ for all $t \in T$. Moreover, int $\Pi_{2}$ is dense in $\Pi_{2}$.

(iii) $\pi \in \operatorname{int} \Pi_{3}$ if and only if $\left(0_{n}, 1\right) \in \operatorname{int} N$. Moreover, int $\Pi_{3}$ is dense in $\Pi_{3}$ if and only if $|T| \geq n+1$.

(iv) int $\Pi_{4}=\emptyset$.

(v) The primal-dual stable parameters form an open and dense subset of $\Pi$.

Proof (i) Since $\Pi_{1}=\Pi_{c}^{P} \cap \Pi_{c}^{D}$, int $\Pi_{1}=\left(\operatorname{int} \Pi_{c}^{P}\right) \cap\left(\operatorname{int} \Pi_{c}^{D}\right)$ and the first statement follows from Lemma 2, parts (i) and (iii).

The density of int $\Pi_{1}$ in $\Pi_{1}$ entails int $\Pi_{1} \neq \emptyset$. If $\pi^{1}=\left(a^{1}, b^{1}, c^{1}\right) \in \operatorname{int} \Pi_{1}$, we have $c^{1} \in \operatorname{int} M_{1}$ and this is only possible if $|T| \geq n$. The converse statement is a particular case of [36, Theorem 1.8].

(ii) Since $\Pi_{2}=\Pi_{C}^{P} \cap \Pi_{I C}^{D}$, int $\Pi_{2}=\left(\operatorname{int} \Pi_{C}^{P}\right) \cap\left(\operatorname{int} \Pi_{I C}^{D}\right)$ and the conclusion follows from statements (i) and (iv) in Lemma 2, taking into account that $a_{t}^{\prime} y>0$ for all $t \in T$ implies the Slater condition. In fact, if $0_{n+1} \notin$ $\operatorname{conv}\left\{\left(a_{t}, b_{t}\right), t \in T\right\}$ we can write $0_{n+1}=\sum_{t \in T} \lambda_{t}\left(a_{t}, b_{t}\right)$ for some $\lambda \in \mathbb{R}_{+}^{(T)}$ such that $\sum_{t \varepsilon T} \lambda_{t}=1$. Then, multiplying by $(y, 0)$ we get $0=\sum_{t \varepsilon T} \lambda_{t}\left(a_{t}^{\prime} y\right)>0$ (contradiction).

Now let $\pi^{\infty}=\left(a^{\infty}, b^{\infty}, c^{\infty}\right) \in \Pi_{2}$. Select an arbitrary $\bar{x} \in F_{\infty}$. By Diagram 2, we have $c^{\infty} \notin M_{\infty}$, which is a closed convex cone. By the separation theorem, there exists $d \in \mathbb{R}^{n}$ such that $d^{\prime} c^{\infty}<0$ and $d^{\prime} z \geq 0$ for all $z \in M_{\infty}$. For $r \in \mathbb{N}$ we define $a_{t}^{r}=a_{t}^{\infty}+\frac{d}{r}$ and $b_{t}^{r}=b_{t}^{\infty}+\frac{d^{\prime} \bar{x}-1}{r}$ for all $t \in T$, and $c^{r}=c^{\infty}$. Obviously, the sequence $\left\{\pi^{r}\right\}_{r=1}^{\infty}$ such that $\pi^{r}:=\left(a^{r}, b^{r}, c^{r}\right)$ converges to $\pi^{\infty}$. Moreover, $d^{\prime} a_{t}^{r}=d^{\prime} a_{t}^{\infty}+\frac{\|d\|^{2}}{r}>0$ for all $t \in T$, and $d^{\prime} c^{r}=d^{\prime} c^{\infty}<0$. Then $\left\{\pi^{r}\right\}_{r=1}^{\infty} \subset \operatorname{int} \Pi_{2}$. Thus int $\Pi_{2}$ is dense in $\Pi_{2}$.

(iii) In LP, we have $\Pi_{3}=\left(\Pi \backslash \Pi_{c}^{P}\right) \cap \Pi_{c}^{D}$ and the conclusion follows again from Lemma 2 , statements (ii) and (iii) (observe that $\left(0_{n}, 1\right) \in \operatorname{int} N$ guarantees $M=\mathbb{R}^{n}$ ).

The density of int $\Pi_{3}$ in $\Pi_{3}$ entails int $\Pi_{3} \neq \emptyset$. If $\pi^{1}=\left(a^{1}, b^{1}, c^{1}\right) \in$ int $\Pi_{3}$, we have $\left(0_{n}, 1\right) \in \operatorname{int} N_{1}$ and so, $|T| \geq n+1$. Conversely, assume that $|T| \geq n+1$. Let $\pi^{\infty}=\left(a^{\infty}, b^{\infty}, c^{\infty}\right) \in \Pi_{3}$. Since

$$
\left(0_{n}, 1\right) \in N_{\infty}=\text { cone }\left\{\left(a_{t}^{\infty}, b_{t}^{\infty}\right), t \in T\right\},
$$

given $r=1,2, \ldots$, by Lemma 1 (iii), there exists a set $\left\{\left(a_{t}^{r}, b_{t}^{r}\right), t \in T\right\} \subset \mathbb{R}^{n+1}$ such that

$$
\left\|\left(\begin{array}{c}
a_{t}^{\infty} \\
b_{t}^{\infty}
\end{array}\right)-\left(\begin{array}{c}
a_{t}^{r} \\
b_{t}^{r}
\end{array}\right)\right\|_{\infty}<\frac{1}{r}
$$


and

$$
\left(0_{n}, 1\right) \in \operatorname{int} \text { cone }\left\{\left(a_{t}^{r}, b_{t}^{r}\right), t \in T\right\} .
$$

Defining $c^{r}=c^{\infty}$ and $\pi^{r}=\left(a^{r}, b^{r}, c^{r}\right)$ for all $r=1,2, \ldots$, we have $\lim _{r} \pi^{r}=\pi^{\infty}$ and $\left\{\pi^{r}\right\}_{r=1}^{\infty} \subset \operatorname{int} \Pi_{3}$.

(iv) First we assume $\pi=(a, b, c) \in \operatorname{int} \Pi_{4}$. By Lemma 2, statements (ii) and (iv), $\left(0_{n}, 1\right) \in \operatorname{int} N$ and there exists $y \in \mathbb{R}^{n}$ such that $c^{\prime} y<0$ and $a_{t}^{\prime} y>0$ for all $t \in T$. Let $\lambda \in \mathbb{R}_{+}^{(T)}$ be such that

$$
\left(\begin{array}{c}
0_{n} \\
1
\end{array}\right)=\sum_{t \varepsilon T} \lambda_{t}\left(\begin{array}{c}
a_{t} \\
b_{t}
\end{array}\right)
$$

Since $\sum_{t \varepsilon T} \lambda_{t} b_{t}=1, \sum_{t \varepsilon T} \lambda_{t}>0$. Multiplying by $y$ both members of the equation $\sum_{t \varepsilon T} \lambda_{t} a_{t}=0_{n}$ (derived from $\left.(7)\right)$ we get $\sum_{t \in T} \lambda_{t}\left(a_{t}^{\prime} y\right)=0$, but this is impossible. Consequently, int $\Pi_{4}=\emptyset$.

(v) Let $\Omega$ be the class of primal-dual stable parameters. From the proof of statements (i)-(iv),

$$
\Omega= \begin{cases}\operatorname{int} \Pi_{2}, & \text { if }|T|<n, \\ \left(\operatorname{int} \Pi_{1}\right) \cup\left(\operatorname{int} \Pi_{2}\right), & \text { if }|T|=n, \\ \left(\operatorname{int} \Pi_{1}\right) \cup\left(\operatorname{int} \Pi_{2}\right) \cup\left(\operatorname{int} \Pi_{3}\right), & \text { if }|T|>n,\end{cases}
$$

so that $\Omega$ is open. Finally we prove the density of $\Omega$ in $\Pi$ through a discussion based on the cardinality of $T$.

(a) Let $|T|<n$. Since $\Omega=\operatorname{int} \Pi_{2}$ is dense in $\Pi_{2}$, we have just to prove that $\Pi_{1} \cup \Pi_{3} \cup \Pi_{4} \subset \mathrm{cl} \Pi_{2}$.

If $\pi^{\infty}=\left(a^{\infty}, b^{\infty}, c^{\infty}\right) \in \Pi_{1}$, we have by Diagram $2,\left(0_{n}, 1\right) \notin N_{\infty}$ and $c^{\infty} \in M_{\infty}$. Since $\operatorname{dim} M_{\infty} \leq|T|<n, c^{\infty} \in \operatorname{bd} M_{\infty}$ and there exists a sequence $\left\{c^{r}\right\}_{r=1}^{\infty} \subset \mathbb{R}^{n}$ such that $c^{r} \notin M_{\infty}$ and $\left\|c^{r}-c^{\infty}\right\|_{\infty}<\frac{1}{r}, r=1,2, \ldots$ Defining $\pi^{r}:=\left(a^{\infty}, b^{\infty}, c^{r}\right) \in \Pi$, we have $\left(0_{n}, 1\right) \notin N_{r}=N_{\infty}$ and $c^{r} \notin M_{r}=$ $M_{\infty}$, so that $\pi^{r} \in \Pi_{2}, r=1,2, \ldots$ Then $\pi^{\infty}=\lim _{r} \pi^{r}$, so that $\Pi_{1} \subset \operatorname{cl} \Pi_{2}$.

Alternatively, if $\pi^{\infty} \in \Pi_{3} \cup \Pi_{4}$, then $\left(0_{n}, 1\right) \in N_{\infty}$, with $\operatorname{dim} N_{\infty} \leq$ $|T|<n$. By Lemma 1(ii), there exists $\left(a^{r}, b^{r}\right) \in \mathcal{C}(T)^{n+1}$ such that $\left(0_{n}, 1\right)$ $\notin$ cone $\left\{\left(a_{t}^{r}, b_{t}^{r}\right), t \in T\right\}$ and $\left\|\left(a^{r}, b^{r}\right)-\left(a^{\infty}, b^{\infty}\right)\right\|_{\infty}<\frac{1}{r}, r=1,2, \ldots$ Defining $\pi^{r}:=\left(a^{r}, b^{r}, c^{\infty}\right) \in \Pi$, we have $\left(0_{n}, 1\right) \notin N_{r}$, i.e., $\pi^{r} \in \Pi_{1} \cup \Pi_{2} \subset \operatorname{cl} \Pi_{2}$, $r=1,2, \ldots$ Thus $\Pi_{3} \cup \Pi_{4} \subset \operatorname{cl} \Pi_{2}$.

(b) Let $|T|=n$. Since $\Omega=\left(\operatorname{int} \Pi_{1}\right) \cup\left(\operatorname{int} \Pi_{2}\right)$ is dense in $\Pi_{1} \cup \Pi_{2}$, we have just to prove that $\Pi_{3} \cup \Pi_{4} \subset \mathrm{cl}\left(\Pi_{1} \cup \Pi_{2}\right)$. In fact, given $\pi^{\infty}=$ $\left(a^{\infty}, b^{\infty}, c^{\infty}\right) \in \Pi_{3} \cup \Pi_{4}$, we have $\left(0_{n}, 1\right) \in N_{\infty}$, with $\operatorname{dim} N_{\infty} \leq|T|<n+1$. The rest of the proof is as in the second case of (a).

(c) Now we assume $|T| \geq n+1$. Since $\Omega=\bigcup_{i=1}^{3} \operatorname{int} \Pi_{i}$, and int $\Pi_{i}$ is dense in $\Pi_{i}, i=1,2,3$, it is sufficient to show that $\Pi_{4} \subset \mathrm{cl}\left(\bigcup_{i=1}^{3} \Pi_{i}\right)$, but this is a consequence of int $\Pi_{4}=\emptyset$.

The proof is complete. 
Statement (v) in Theorem 1 means that the set of primal-dual unstable parameters is small in a topological sense (and so $\Pi_{4}$ is also small). The next example shows that an element of $\Pi_{4}$ can be approached by primal-dual stable parameters of the three remaining duality states.

Example 1 Let $|T| \geq n+1$ and let $t_{1}, \ldots, t_{n+1}$ be different elements of $T$. Let $\pi=(a, b, c) \in \Pi$ be such that

$$
a_{t}:=\left\{\begin{array}{l}
0_{n}, t \neq t_{n+1}, \\
e_{1}, t=t_{n+1},
\end{array} \quad b_{t}:=\left\{\begin{array}{l}
1, t \neq t_{n+1} \\
0, t=t_{n+1}
\end{array}\right.\right.
$$

and $c=e$. Since $\left(0_{n}, 1\right) \in N$ and $c \notin M, \pi \in \Pi_{4}$. We give three sequences of the form $\pi^{r}=\left(a^{r}, b, c\right), r=1,2, \ldots$, such that $\lim _{r} \pi^{r}=\pi$, each of the sequences contained in a different set int $\Pi_{i}, i=1,2,3$. We define $a_{t}^{r}$ in the three cases as follows:

(i) $\left\{\begin{array}{l}\frac{e_{i}}{r}, t=t_{i}, i=1, \ldots, n, \\ e_{1}, t=t_{n+1}, \\ \frac{e}{r}, \text { otherwise. }\end{array}\right.$ (ii) $\left\{\begin{array}{l}\frac{e_{1}}{r}, t \neq t_{n+1}, \\ e_{1}, t=t_{n+1} .\end{array}\right.$ (iii) $\left\{\begin{array}{l}-\frac{e}{r}, \quad t=t_{1}, \\ \frac{e_{i}}{r}, t=t_{2}, \ldots, t_{n}, \\ e_{1}, \quad t=t_{n+1}, \\ 0_{n}, \quad \text { otherwise. }\end{array}\right.$

We get the conclusion from Theorem 1, taking into account that:

Case (i): $2 r e$ is a Slater element for $\pi^{r}$ and $c^{r}=e \in \operatorname{int} M_{r}=\operatorname{int} \mathbb{R}_{+}^{n}$;

Case (ii): $2 r e$ is a Slater element for $\pi^{r}$ and $y^{r}:=(1,-(r+1), \ldots,-(r+1)) \in$ $\mathbb{R}^{n}$ satisfies $c^{\prime} y^{r}=1-(n-1)(r+1)<0$, and $\left(a_{t}^{r}\right)^{\prime} y^{r}=\frac{1}{r}>0$ if $t \neq t_{n+1}$ and $\left(a_{t}^{r}\right)^{\prime} y^{r}=1$ otherwise; and

Case (iii): $\frac{1}{n+1}\left(\begin{array}{c}-\frac{e}{r} \\ 1\end{array}\right)+\frac{1}{n+1} \sum_{i=2}^{n}\left(\begin{array}{c}\frac{e_{i}}{r} \\ 1\end{array}\right)+\frac{1}{r(n+1)}\left(\begin{array}{c}e_{1} \\ 0\end{array}\right)=\frac{n}{n+1}\left(\begin{array}{c}0_{n} \\ 1\end{array}\right)$, so that $\left(0_{n}, 1\right) \in \operatorname{int} N_{r}$

\section{Primal-dual stability in continuous LSIP}

Let $\Pi$ be the space of parameters corresponding to a given triple $\pi:=(a, b, c)$ such that $T$ is an infinite compact Hausdorff topological space and $n \geq 2$. The next two lemmas provide the LSIP counterpart of Diagram 2, allowing us the classification of $\pi$ in terms of the associated moment cones.

Lemma 3 Let $\pi \in \Pi_{c}^{P}$. Then $v^{P}(\pi) \neq-\infty$ if and only if

$$
(\{c\} \times \mathbb{R}) \cap \operatorname{cl} N \neq \emptyset .
$$

Proof $v^{P}(\pi) \neq-\infty$ if and only if there exists $\alpha \in \mathbb{R}$ such that $c^{\prime} x \geq \alpha$ for all $x \in F$, i.e., $(c, \alpha) \in \operatorname{cl} K$.

If (8) holds, then there exists $\alpha \in \mathbb{R}$ such that $(c, \alpha) \in \operatorname{cl} N \subset \operatorname{cl} K$.

Conversely, assume that $(c, \alpha) \in \operatorname{cl} K$. Then there exist $\left\{\lambda^{r}\right\}_{r=1}^{\infty} \subset \mathbb{R}_{+}^{(T)}$ and $\left\{\gamma^{r}\right\}_{r=1}^{\infty} \subset \mathbb{R}_{+}$such that

$$
\left(\begin{array}{c}
c \\
\alpha
\end{array}\right)=\lim _{r}\left\{\sum_{t \in T} \lambda_{t}^{r}\left(\begin{array}{c}
a_{t} \\
b_{t}
\end{array}\right)+\gamma^{r}\left(\begin{array}{c}
0_{n} \\
-1
\end{array}\right)\right\} .
$$


If $\left\{\gamma^{r}\right\}_{r=1}^{\infty}$ is unbounded we can assume that $\lim _{r} \gamma^{r}=+\infty$, with $\gamma^{r}>0$, $r=1,2, \ldots$ From (9) we get $c=\lim _{r}\left(\sum_{t \in T} \lambda_{t}^{r} a_{t}\right)$, so that

$$
\lim _{r}\left(\sum_{t \in T}\left(\gamma^{r}\right)^{-1} \lambda_{t}^{r} a_{t}\right)=0_{n}
$$

Now we define $\alpha_{r}:=\left(\sum_{t \in T} \lambda_{t}^{r} b_{t}\right)-\gamma^{r}, r=1,2, \ldots$ From $(9), \lim _{r} \alpha_{r}=\alpha$. Then $\lim _{r}\left(\gamma^{r}\right)^{-1} \alpha_{r}=0$, so that

$$
\lim _{r}\left(\sum_{t \in T}\left(\gamma^{r}\right)^{-1} \lambda_{t}^{r} b_{t}\right)=1
$$

From (10) and (11) we get $\left(0_{n}, 1\right) \in \operatorname{cl} N$, in contradiction with $\pi \in \Pi_{c}^{P}$.

Thus $\left\{\gamma^{r}\right\}_{r=1}^{\infty}$ is bounded and so it contains a convergent subsequence. We can assume that $\lim _{r} \gamma^{r}=\gamma \in \mathbb{R}_{+}$. Since $\lim _{r} \gamma^{r}\left(0_{n},-1\right)=\left(0_{n},-\gamma\right),(9)$ yields

$$
\left(\begin{array}{c}
c \\
\alpha+\gamma
\end{array}\right)=\lim _{r}\left\{\sum_{t \in T} \lambda_{t}^{r}\left(\begin{array}{c}
a_{t} \\
b_{t}
\end{array}\right)\right\} \in \operatorname{cl} N
$$

and so (8) holds.

Corollary 1 (i) $\pi \in \Pi_{2}$ if and only if $\left(0_{n}, 1\right) \notin \operatorname{cl} N$ and $(\{c\} \times \mathbb{R}) \cap \operatorname{cl} N=$ $\emptyset$.

(ii) $\pi \in \Pi_{5}$ if and only if $c \notin M,\left(0_{n}, 1\right) \notin \mathrm{cl} N$ and $(\{c\} \times \mathbb{R}) \cap \mathrm{cl} N \neq \emptyset$.

Proof It is straightforward consequence of Lemma 3.

Lemma 4 Let $\pi \in \Pi_{c}^{D}$. Then $v^{D}(\pi) \neq+\infty$ if and only if

$$
\{c\} \times \mathbb{R} \nsubseteq K=N+\mathbb{R}_{+}\left\{\left(0_{n},-1\right)\right\} .
$$

Proof It is immediate consequence of the geometric interpretation of $D$ : $v^{D}(\pi)=\sup \{\alpha \mid(c, \alpha) \in N\}$.

Corollary 2 (i) $\pi \in \Pi_{3}$ if and only if $\{c\} \times \mathbb{R} \subset N+\mathbb{R}_{+}\left\{\left(0_{n},-1\right)\right\}$. (ii) $\pi \in \Pi_{6}$ if and only if $\left(0_{n}, 1\right) \in \operatorname{cl} N, c \in M$ and $\{c\} \times \mathbb{R} \nsubseteq N+$ $\mathbb{R}_{+}\left\{\left(0_{n},-1\right)\right\}$.

Proof (i) The direct statement is an immediate consequence of Lemma 4 (observe that $\{c\} \times \mathbb{R} \subset N+\mathbb{R}_{+}\left\{\left(0_{n},-1\right)\right\}$ ensures that $r^{-1}(c, r) \in N$, for all $r=1,2, \ldots$, so that $\left.\left(0_{n}, 1\right) \in \operatorname{cl} N\right)$. Conversely, assume $\{c\} \times \mathbb{R} \subset K$. Obviously, $c \in M$ and Lemma 4 yields $\pi \in \Pi_{3}$.

(ii) It is also a straightforward consequence of Lemma 4. 
As a consequence of statement (ii) in Corollary 2 , if $\pi \in \Pi_{6}$ then $\left(0_{n}, 1\right) \notin$ $N$ and so $N$ is non-closed. In fact, $\left(0_{n}, 1\right) \in N$ and $c \in M$ imply the existence of $\alpha \in \mathbb{R}$ such that $(c, \gamma) \in N$ for all $\gamma \geq \alpha$, so that $\{c\} \times \mathbb{R} \subset K$.

Diagram 3 summarizes the characterization of the duality states $\Pi_{i}, i=$ $1, \ldots, 6$, in terms of $M$ and $N$. There $\rceil(\mathrm{j})$ stands for the negation of statement (j).

\begin{tabular}{|c|c|c|c|c|}
\hline & \multirow{2}{*}{$\left(0_{n}, 1\right) \in \operatorname{cl} N$} & \multicolumn{2}{|c|}{$\left(0_{n}, 1\right) \notin \operatorname{cl} N$} \\
\hline & & & (8) & $7(8)$ \\
\hline \multicolumn{2}{|c|}{$\overline{c c \notin M}$} & 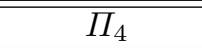 & 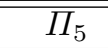 & $\overline{\Pi_{2}}$ \\
\hline$c \in M$ & $\begin{array}{c}(12) \\
7(12)\end{array}$ & $\begin{array}{l}\Pi_{6} \\
\Pi_{3}\end{array}$ & \multicolumn{2}{|c|}{$\Pi_{1}$} \\
\hline
\end{tabular}

Diagram 3

The next example shows that $\Pi_{i} \neq \emptyset, i=4,5,6$, provided the compact set $T$ is infinite.

Example 2 In the proof of [19, Theorem 6.4(iii)] we have shown the existence of a sequence of non-repeated indices $\left\{t_{r}\right\}_{r=1}^{\infty} \subset T$, and a scalar mapping $\varphi \in \mathcal{C}(T)$ such that $\varphi\left(t_{r}\right)=2^{1-r}, r=1,2, .$. , and $0 \leq \varphi(t) \leq 1$ for all $t \in T$. Then $\pi^{1}:=\left(\varphi, 0_{n-1}, \varphi^{2}, e_{2}\right) \in \Pi_{4}$ because $\left(0_{n}, 1\right) \in \operatorname{cl} N_{1}$ and $c^{1} \notin M_{1}$, $\pi^{2}:=\left(\varphi, \varphi^{2}, 0_{n-1}, e_{1}\right) \in \Pi_{5}$, by Corollary 1 , and $\pi^{3}:=\left(\varphi, 0_{n-1}, \varphi^{\frac{1}{2}}, 0_{n}\right) \in$ $\Pi_{6}$, by Corollary 2 .

Theorem 2 The following statements are true:

(i) $\pi \in \operatorname{int} \Pi_{1}$ if and only if Slater condition holds and $c \in \operatorname{int}$ M. Moreover, int $\Pi_{1}$ is dense in $\Pi_{1}$.

(ii) $\pi \in \operatorname{int} \Pi_{2}$ if and only if there exists $y \in \mathbb{R}^{n}$ such that

$$
c^{\prime} y<0 \text { and } a_{t}^{\prime} y>0 \text { for all } t \in T \text {. }
$$

Moreover, int $\Pi_{2}$ is dense in $\Pi_{2}$.

(iii) $\pi \in \operatorname{int} \Pi_{3}$ if and only if $\left(0_{n}, 1\right) \in \operatorname{int} N$. Moreover, int $\Pi_{3}$ is dense in $\Pi_{3}$.

(iv) int $\Pi_{i}=\emptyset, i=4,5,6$.

(v) The class of primal-dual stable parameters is an open and dense subset of $\Pi$.

Proof (i) The proof is the same as in Theorem 1(i).

(ii) Since $\Pi_{2} \subset \Pi_{c}^{P} \cap\left(\Pi \backslash \Pi_{c}^{D}\right)$, the direct statement of the first part is the same as in Theorem 1(ii). For the converse statement we assume the existence of $y \in \mathbb{R}^{n}$ satisfying (13), which implies the Slater condition. Since (13), for the same vector $y$, defines an open subset of $\Pi$ by the continuity assumption, there exists $\varepsilon>0$ such that $\pi^{1}:=\left(a^{1}, b^{1}, c^{1}\right) \in \Pi$ also satisfies (13) if $d\left(\pi^{1}, \pi\right)<\varepsilon$. Recall that the Slater condition of $\pi^{1}$ guarantees that $N_{1}$ is closed and $\pi^{1} \in \Pi_{c}^{P}$.

If $\left(\left\{c^{1}\right\} \times \mathbb{R}\right) \cap N_{1} \neq \emptyset$, there exists $\alpha \in \mathbb{R}$ and $\lambda \in \mathbb{R}_{+}^{(T)}$ such that

$$
\left(\begin{array}{c}
c^{1} \\
\alpha
\end{array}\right)=\sum_{t \in T} \lambda_{t}\left(\begin{array}{c}
a_{t}^{1} \\
b_{t}^{1}
\end{array}\right) .
$$


Multiplying both members of $(14)$ by $(y, 0)$, we get the following contradiction:

$$
0>\left(c^{1}\right)^{\prime} y=\sum_{t \in T} \lambda_{t}\left(a_{t}^{1}\right)^{\prime} y \geq 0 .
$$

Consequently, $\pi^{1}$ violates $(8)$ and so $v^{P}\left(\pi^{1}\right)=-\infty$, i.e., $\pi^{1} \in \Pi_{2}$. Hence $\pi \in \operatorname{int} \Pi_{2}$.

The proof of the density of int $\Pi_{2}$ in $\Pi_{2}$ is also similar to the corresponding part of Theorem 1(ii). The only difficulty comes from the fact that $M_{\infty}$ could be non-closed. Nevertheless, since $c^{\infty} \notin M_{\infty}$, in the worst case $c^{\infty} \in \operatorname{bd} M_{\infty}$ and so there exists a sequence $\left\{c^{r}\right\}_{r=1}^{\infty} \subset \mathbb{R}^{n} \backslash \mathrm{cl} M_{\infty}$ such that $\lim _{r} c^{r}=c^{\infty}$. For each $r \in \mathbb{N}$ there exists $d^{r} \in \mathbb{R}^{n}$ such that $\left\|d^{r}\right\|=1,\left(c^{r}\right)^{\prime} d^{r}<0$ and $\left(a_{t}^{\infty}\right)^{\prime} d^{r} \geq 0$ for all $t \in T$. Replacing $d$ with $d^{r}$ in the definition of $\pi^{r} \in \Pi$, we get the same conclusion with the same argument.

(iii) First we prove that $\pi \in \operatorname{int} \Pi_{3}$ ensures

$$
(\{c\} \times \mathbb{R}) \cup\left\{\left(0_{n}, 1\right)\right\} \subset \operatorname{int} K .
$$

Assume that (15) fails. Since $\Pi_{3} \subset \Pi \backslash \Pi_{c}^{P},\left(0_{n}, 1\right) \in \operatorname{int} N \subset \operatorname{int} K$ according to Lemma 2(ii). Then we must have $\{c\} \times \mathbb{R} \nsubseteq$ int $K$. Moreover, by Corollary $2,\{c\} \times \mathbb{R} \subset K$. Thus there exists $\alpha \in \mathbb{R}$ such that $(c, \alpha) \in \operatorname{bd} K$. By the supporting hyperplane theorem for cones there exists $(d, \gamma) \in \mathbb{R}^{n+1} \backslash$ $\left\{0_{n+1}\right\}$ such that

$$
(d, \gamma)\left(\begin{array}{c}
c \\
\alpha
\end{array}\right)=0
$$

and

$$
(d, \gamma)\left(\begin{array}{c}
x \\
x_{n+1}
\end{array}\right) \geq 0 \text { for all }\left(\begin{array}{c}
x \\
x_{n+1}
\end{array}\right) \in K
$$

From (17) we get

$$
a_{t}^{\prime} d+\gamma b_{t} \geq 0, \text { for all } t \in T, \text { and } \gamma \leq 0 .
$$

According to (18) two cases can arise.

If $\gamma<0$ then $-\gamma^{-1} d \in F$ and so $\pi \in \Pi_{c}^{P}$, contradicting $\pi \in \operatorname{int} \Pi_{3}$.

Hence $\gamma=0$, so that $d \neq 0_{n}$ and $c^{\prime} d=0$, by (16).

Consider the sequence $\pi^{r}:=\left(a, b, c-\frac{d}{r}\right), r=1,2, \ldots$ If $\pi^{r} \in \Pi_{3}$ then, by Corollary $2,\left(c-\frac{d}{r}, 0\right) \in K_{r}=K$ and, by (17), we get the following contradiction: $0 \leq d^{\prime}\left(c-\frac{d}{r}\right)=-\frac{\|d\|^{2}}{r}<0$.

Thus $\left\{\pi^{r}\right\}_{r=1}^{\infty} \subset \Pi \backslash \Pi_{3}$ and $\lim _{r} \pi^{r}=\pi$, in contradiction with $\pi \in \operatorname{int} \Pi_{3}$.

Conversely, assume that $\pi$ satisfies (15). Observe that we can write $K=$ cone $\left\{\left(a_{t}, b_{t}\right), t \in \widetilde{T}\right\}$, where $\widetilde{T}=T \cup\{s\}$ is a compact Hausdorff topological space, $s$ is an isolated point of $\widetilde{T},\left(a_{s}, b_{s}\right)=\left(0_{n},-1\right)$ and $(a, b) \in \mathcal{C}(\widetilde{T})^{n+1}$. Since $\left\{(c, 0),\left(0_{n}, 1\right)\right\} \subset$ int $K$, by Lemma $1(\mathrm{i})$, there exists $\varepsilon>0$ such that $\left\{\left(c^{1}, 0\right),\left(0_{n}, 1\right)\right\} \subset \operatorname{int} K_{1}$ for all $\pi^{1} \in \Pi$ such that $d\left(\pi^{1}, \pi\right)<\varepsilon$. For such a parameter $\pi^{1}$ we have $\pm\left(0_{n}, 1\right) \in K_{1}$ and $\left(c^{1}, 0\right) \in K_{1}$, and this entails $\left\{c^{1}\right\} \times \mathbb{R} \subset K_{1}$, i.e., $\pi^{1} \in \Pi_{3}$. Hence $\pi \in \operatorname{int} \Pi_{3}$ by Corollary 2 . 
It remains to be proved that $(15)$ is equivalent to $\left(0_{n}, 1\right) \in \operatorname{int} N$.

If (15) holds, $\pi \in \Pi_{3} \subset \Pi \backslash \Pi_{c}^{P}$, so that $\left(0_{n}, 1\right) \in \operatorname{cl} N$. If $\left(0_{n}, 1\right) \notin \operatorname{int} N$, then $\left(0_{n}, 1\right) \in \operatorname{bd} N$ and there exists a supporting hyperplane to $N$ at $\left(0_{n}, 1\right)$ which turns out to be also supporting hyperplane to $K$ at $\left(0_{n}, 1\right)$, so that $\left(0_{n}, 1\right) \in \operatorname{bd} K$ in contradiction with $(15)$.

Now we assume that $\left(0_{n}, 1\right) \in \operatorname{int} N$. Since $\lim _{\rho \rightarrow+\infty}\left(\frac{c}{\rho}, 1\right)=\left(0_{n}, 1\right) \in$ $\operatorname{int} N,\left(\frac{c}{\rho}, 1\right) \in \operatorname{int} N$ for $\rho$ big enough such that $\alpha<\rho$. In such a case, $(c, \rho) \in \operatorname{int} N$ and we have

$$
\left(\begin{array}{c}
c \\
\alpha
\end{array}\right)=\left(\begin{array}{c}
c \\
\rho
\end{array}\right)+(\rho-\alpha)\left(\begin{array}{c}
0_{n} \\
-1
\end{array}\right) \in \operatorname{int} N+(\rho-\alpha)\left(\begin{array}{c}
0_{n} \\
-1
\end{array}\right) \subset \operatorname{int} K,
$$

because $\operatorname{int} N \subset \operatorname{int} K$ and $\left(0_{n},-1\right)$ is a recession direction of $K$. Hence $\{c\} \times \mathbb{R} \subset \operatorname{int} K$. Then (15) holds.

The proof of the second statement in (iii) is the same as in Theorem 1 , observing that the mappings $\left(a^{r}, b^{r}\right): T \rightarrow \mathbb{R}^{n+1}$ can be chosen continuous on $T$ according to Lemma 1 (iii).

(iv) The proof of int $\Pi_{4}=\emptyset$ is the same as in Theorem 1 . Next we prove that int $\Pi_{5}=\operatorname{int} \Pi_{6}=\emptyset$.

Assume $\pi \in \operatorname{int} \Pi_{5}$. Then $\pi \in \operatorname{int} \Pi_{c}^{P}$ and the Slater condition holds, so that $N$ is closed. Then Corollary 1 yields $(\{c\} \times \mathbb{R}) \cap N \neq \emptyset$ and so, $c \in M$. This contradicts $\pi \in \Pi_{5}$. Hence, int $\Pi_{5}=\emptyset$.

Finally, assume $\pi \in$ int $\Pi_{6}$. Since $\Pi_{6} \subset \Pi \backslash \Pi_{c}^{P}$, we have $\left(0_{n}, 1\right) \in \operatorname{int} N$ by Lemma 2(ii). On the other hand, since $\Pi_{6} \subset \Pi_{c}^{D}$, we have $c \in M$ so that there exists $\alpha \in \mathbb{R}$ such that $(c, \alpha) \in N$. Given $\gamma \in \mathbb{R}$ two cases are possible:

If $\gamma \leq \alpha$, then

$$
\left(\begin{array}{c}
c \\
\gamma
\end{array}\right)=\left(\begin{array}{c}
c \\
\alpha
\end{array}\right)+(\alpha-\gamma)\left(\begin{array}{c}
0_{n} \\
-1
\end{array}\right) \in K
$$

Alternatively, if $\gamma>\alpha$, then

$$
\left(\begin{array}{c}
c \\
\gamma
\end{array}\right)=\left(\begin{array}{c}
c \\
\alpha
\end{array}\right)+(\gamma-\alpha)\left(\begin{array}{c}
0_{n} \\
1
\end{array}\right) \in N \subset K
$$

In both cases $(c, \gamma) \in K$. Since $\pi$ satisfies $\{c\} \times \mathbb{R} \subset K, \pi \in \Pi_{3}$ by Corollary 2 , which contradicts $\pi \in \operatorname{int} \Pi_{6}$.

(v) We must prove that the set of primal-dual stable parameters $\Omega:=$ $\bigcup_{i=1}^{3} \operatorname{int} \Pi_{i}$ (which is obviously open) is dense in $\Pi$. According to (i)-(iv), it is sufficient to prove that int $\left(\bigcup_{i=4}^{6} \Pi_{i}\right)=\emptyset$.

First we prove that int $\left(\Pi_{5} \cup \Pi_{6}\right)=\emptyset$.

Assume the contrary, i.e., $V:=\operatorname{int}\left(\Pi_{5} \cup \Pi_{6}\right) \neq \emptyset$. According to statement (iv), since $V$ is open and $V \subset \Pi_{5} \cup \Pi_{6}$ we must have $V \cap \Pi_{5} \neq \emptyset \neq V \cap \Pi_{6}$. 
Let $\pi^{1}:=\left(a^{1}, b^{1}, c^{1}\right) \in V \cap \Pi_{6}$ and let $\varepsilon>0$ be such that $\pi^{2} \in V$ if $d\left(\pi^{2}, \pi^{1}\right)<\varepsilon$. We prove now the existence of $\pi^{2}=\left(a^{2}, b^{2}, c^{2}\right) \in V$ such that $c^{1} \in \operatorname{int} M_{2}$ discussing two possible cases.

Let $c^{1}=0_{n}$. Since $\pi^{1} \in \Pi_{6}$, by Corollary $2,\left\{0_{n}\right\} \times \mathbb{R} \nsubseteq K_{1}$ and so $\left(0_{n}, 1\right) \in\left(\operatorname{cl} K_{1}\right) \backslash K_{1}$ because $\left(0_{n},-1\right) \in K_{1}$ and $\left(0_{n}, 1\right) \in \operatorname{cl} K_{1}$. Since $\left(0_{n}, 1\right) \in \operatorname{cl} N_{1}$ and $N_{1} \subset K_{1}$, we have $\left(0_{n}, 1\right) \in\left(\operatorname{cl} N_{1}\right) \backslash N_{1}$, so that $N_{1}$ is non-closed and $0_{n+1} \in \operatorname{conv}\left\{\left(\begin{array}{c}a_{t}^{1} \\ b_{t}^{1}\end{array}\right), t \in T\right\}$. By Lemma 1(iv) there exists $\left(a^{2}, b^{2}\right) \in \mathcal{C}(T)^{n+1}$ such that $0_{n+1} \in \operatorname{int}$ cone $\left\{\left(\begin{array}{c}a_{t}^{2} \\ b_{t}^{2}\end{array}\right), t \in T\right\}$ and $\left\|\left(a^{2}, b^{2}\right)-\left(a^{1}, b^{1}\right)\right\|_{\infty}<\varepsilon$. Then $c^{1}=0_{n} \in \operatorname{int} c o n e\left\{a_{t}^{2}, t \in T\right\}$ and so $\pi^{2}:=\left(a^{2}, b^{2}, c^{1}\right) \in V$.

Alternatively, ssume that $c^{1} \neq 0_{n}$. Since $c^{1} \in M_{1}=$ cone $\left\{a_{t}^{1}, t \in T\right\}$, by Lemma 1 (iii) there exists $a^{2} \in \mathcal{C}(T)^{n}$ such that $\left\|a^{2}-a^{1}\right\|_{\infty}<\varepsilon$ and $c^{1} \in \operatorname{int} M_{2}=$ int cone $\left\{a_{t}^{2}, t \in T\right\}$. Obviously, $\pi^{2}:=\left(a^{2}, b^{1}, c^{1}\right) \in V$.

On the other hand, by Lemma1(i), there exists $\delta>0$ such that $c^{3} \in$ int cone $\left\{a_{t}^{3}, t \in T\right\}$ for all $c^{3} \in \mathbb{R}^{n}$ and $a^{3} \in \mathcal{C}(T)^{n}$ such that $\left\|c^{3}-c^{1}\right\|_{\infty}<\delta$ and $\left\|a^{3}-a^{2}\right\|_{\infty}<\delta$. Then we have $c^{3} \in \operatorname{int} M_{3}$ for all parameter $\pi^{3}:=$ $\left(a^{3}, b^{3}, c^{3}\right) \in \Pi$ such that $d\left(\pi^{3}, \pi^{2}\right)<\delta$. Consider the open set

$$
U:=\left\{\pi^{3} \in V \mid d\left(\pi^{3}, \pi^{2}\right)<\delta\right\} .
$$

Since $V$ is open and $\pi^{2} \in V, U \neq \emptyset$. Moreover, if $\pi^{3} \in U$, then $c^{3} \in \operatorname{int} M_{3}$ and so $\pi^{3} \notin \Pi_{5}$. On the other hand, $\pi^{3} \in V \subset \Pi_{5} \cup \Pi_{6}$, so that necessarily $\pi^{3} \in \Pi_{6}$. We have shown that $U \subset \Pi_{6}$, so that int $\Pi_{6} \neq \emptyset$ in contradiction with (iv).

We complete the proof showing that $\operatorname{int}\left(\Pi_{4} \cup \Pi_{5} \cup \Pi_{6}\right)=\emptyset$.

We assume again the contrary. Let $W:=\operatorname{int}\left(\Pi_{4} \cup \Pi_{5} \cup \Pi_{6}\right) \neq \emptyset$. If $W \cap \Pi_{4}=\emptyset$, we must have $W \subset \Pi_{5} \cup \Pi_{6}$ because $W \subset \Pi_{4} \cup \Pi_{5} \cup \Pi_{6}$. Since this contradicts $\operatorname{int}\left(\Pi_{5} \cup \Pi_{6}\right)=\emptyset$, we have $W \cap \Pi_{4} \neq \emptyset$.

Let $\pi^{1}:=\left(a^{1}, b^{1}, c^{1}\right) \in W \cap \Pi_{4}$. Then $\left(0_{n}, 1\right) \in \operatorname{cl} N_{1}$ and there exists $\varepsilon>0$ such that $d\left(\pi^{2}, \pi^{1}\right)<\varepsilon$ entails $\pi^{2} \in W$. Two cases are possible:

If $0_{n+1} \notin \operatorname{conv}\left\{\left(a_{t}^{1}, b_{t}^{1}\right), t \in T\right\}$, the cone $N_{1}$ is closed and $\left(0_{n}, 1\right) \in N_{1}$. We take then $\pi^{2}:=\pi^{1}$.

Otherwise, we can write

$$
0_{n+1}=\sum_{t \in T} \lambda_{t}\left(\begin{array}{c}
a_{t}^{1} \\
b_{t}^{1}
\end{array}\right), \sum_{t \in T} \lambda_{t}=1, \lambda \in \mathbb{R}_{+}^{(T)} .
$$

Then we consider $\pi^{2}:=\left(a^{1}, b^{1}+\frac{\varepsilon}{2}, c^{1}\right) \in \Pi$. Since $d\left(\pi^{2}, \pi^{1}\right)=\frac{\varepsilon}{2}, \pi^{2} \in$ $W$. From (19) we get

$$
\frac{\varepsilon}{2}\left(\begin{array}{c}
0_{n} \\
1
\end{array}\right)=\sum_{t \in T} \lambda_{t}\left(\begin{array}{c}
a_{t}^{1} \\
b_{t}^{1}+\frac{\varepsilon}{2}
\end{array}\right) \in N_{2}
$$

so that $\left(0_{n}, 1\right) \in N_{2}$. 
In both cases $\pi^{2} \in W$ and $\left(0_{n}, 1\right) \in N_{2}$. Let $\delta>0$ be such that $\pi^{3} \in W$ if $d\left(\pi^{3}, \pi^{2}\right)<\delta$. By Lemma 1(iii) there exists a continuous mapping $\left(a^{3}, b^{3}\right)$ : $T \rightarrow \mathbb{R}^{n+1}$ such that

$$
\left\|\left(a^{3}, b^{3}\right)-\left(a^{2}, b^{2}\right)\right\|_{\infty}<\delta \text { and }\left(0_{n}, 1\right) \in \operatorname{int} \operatorname{conv}\left\{\left(a_{t}^{3}, b_{t}^{3}\right), t \in T\right\} .
$$

Defining $\pi^{3}:=\left(a^{3}, b^{3}, c^{1}\right) \in \Pi$, we have $\pi^{3} \in W$ and $\left(0_{n}, 1\right) \in \operatorname{int} N_{3}$. By Lemma 1(i) there exists a neighborhood of $\pi^{3}$, say $V, V \subset W$, such that $\left(0_{n}, 1\right) \in \operatorname{int} N_{4}$ for all $\pi^{4} \in V$. In such a case $\pi^{4} \in W \subset \Pi_{5} \cup \Pi_{6}$ and $\pi^{4} \notin \Pi_{5}$ so that $\pi^{4} \in \Pi_{6}$. We have shown that $V \subset \Pi_{6}$, which contradicts (iv).

The proof is complete.

\section{References}

1. Amaya, J., Gómez, J.A.: Strong duality for inexact linear programming problems. Optimization 49, 243-269 (2001)

2. Attouch H., Wets, R.J.B.: Quantitative stability of variational systems: I. The epigraphical distance. Trans. Amer. Math. Soc. 328, 659-729 (1991)

3. Azé, D., Attouch, H., Wets, R.J.B.: Convergence of convex-concave saddle functions: applications to convex programming and mechanics. Ann. Inst. H. Poincaré Anal. Non Linéaire 5, 537-572 (1988)

4. Beer, G.: Topologies on Closed and Closed Convex Sets, Kluwer, Dordrecht, 1993

5. Ben-Israel, A., Charnes, A., Kortanek, K.O.: Duality and asymptotic solvability over cones. Bull. Amer. Math. Soc. 75, 318-324 (1969)

6. Betró, B., Guglielmi, A.: Methods for global prior robustness under generalized moment conditions. In: Robust Bayesian Analysis, Ríos D., Ruggeri F. (eds.), Springer-Verlag, NY, 2000, pp. 273-293

7. Brosowski, B.: Parametric Semi-Infinite Optimization, Verlag Peter Lang, Frankfurt am Main - Bern, 1982

8. Cánovas, M.J., Dontchev, A.L., López, M.A., Parra, J.: Metric regularity of semi-infinite constraint systems. Math. Programming 104B, 329-346 (2005)

9. Cánovas, M.J., López, M.A., Parra, J., Toledo, F.J.: Distance to ill-posedness and the consistency value of linear semi-infinite inequality systems. Math. Program. 103A, 95-126 (2005)

10. Cánovas, M.J., López, M.A., Parra, J., Toledo, F.J.: Distance to solvability/unsolvability in linear optimization. SIAM J. Optim. 16, 629-649 (2006)

11. Dahl, M., Claesson, I., Nordebo, S., Nordholm, S.: Chebyshev optimization of circular arrays. In: Optimization Methods and Applications, Yang X. et al. (eds.), Kluwer, Dordrecht, 2001, pp.309-319

12. Dolgin, Y., Zeheb, E.: Model reduction of uncertain FIR discrete-time systems. IEEE Trans. Circuits and Systems 51, 406-411 (2004)

13. Dolgin, Y., Zeheb, E.: Model reduction of uncertain systems retaining the uncertainty structure. Systems \& Control Letters 54, 771-779 (2005)

14. Fischer, T.: Contributions to semi-infinite linear optimization. Meth. Verf. Math. Phys. 27, 175-199 (1983)

15. Gayá, V.E., López, M.A., Vera de Serio, V.N.: Stability in convex semi-infinite programming and rates of convergence of optimal solutions of discretized finite subproblems. Optimization 52, 693-713 (2003)

16. Goberna, M.A., López, M.A.: Linear Semi-Infinite Optimization, J. Wiley, Chichester, 1998

17. Goberna, M.A., López, M.A., Todorov, M. I.: Stability theory for linear inequality systems. SIAM J. Matrix Anal. Appl. 17, 730-743 (1996)

18. Goberna, M.A., López, M.A., Todorov, M.I.: Stability theory for linear inequality systems II: upper semicontinuity of the solution set mapping. SIAM J. Optim. 7, 1138-1151 (1997) 
19. Goberna, M.A., López, M.A., Todorov, M.I.: On the stability of the feasible set in linear optimization. Set-Valued Analysis 9, 75-99 (2001)

20. Greemberg, H.J., Pierskalla, W.P.: Stability theory for infinitely constrained mathematical programs. J. Optim. Theory Appl. 16, 409-428 (1975)

21. Kallina, C., Williams, A.C.: Linear programming in reflexive spaces. SIAM Rev. 13, 350-376 (1971)

22. Konno, H., Kobayashi, H.: Failure discrimination and rating of enterprises by semi-definite programming. Asia-Pacific Financial Markets 7, 261-273 (2000).

23. Kortanek, K.O., On the 1962-1972 decade of semi-infinite programming: a subjective view. In: Semi-Infinite Programming: Recent Advances, Goberna M.A., López M.A. (eds.), Kluwer, Dordrecht, 2001, pp. 3-41

24. Kortanek, K.O., Medvedev, V.G.: Building and Using Dynamic Interest Rate Models, J. Wiley, Chichester, 2001

25. Kortanek, K.O., Zhang, Q.: Perfect duality in semi-infinite and semidefinite programming. Math. Programming 91A, 127-144 (2001)

26. López, M.A, Vera de Serio, V.N.: Stability of the feasible set mapping in convex semi-infinite programming. In: Semi-Infinite Programming: Recent Advances, Goberna M.A., López M.A. (eds.), Kluwer, Dordrecht, 2001, pp.101-120.

27. Mira, J.A., Mora, G.: Stability of linear inequality systems measured by the Hausdorff metric. Set-Valued Anal. 8, 253-266 (2000)

28. Nordholm, S., Nordberg, J., Claesson, I., Nordebo, S.: Beamforming and interference cancellation for capacity gain in mobile networks. Ann. Oper. Res. 98, 235-253 (2001)

29. Noubiap, R.F., Seidel, W.: An algorithm for calculating Gamma-minimax decision rules under generalized moment conditions. Ann. Stat. 29, 1094-1116 (2001)

30. Robinson, S.M.: Stability theory for systems of inequalities. Part I: Linear systems. SIAM J. Numer. Anal. 12, 754-769 (1975)

31. Rockafellar, R.T., Wets, R.J.B.: Variational Analysis, Springer-Verlag, Berlin, 1998

32. Rubio, J.E.: The optimal control of nonlinear diffusion equations with rough initial data. J. Franklin Inst. 337, 673-690 (2000)

33. Rubio, J.E.: Optimal control problems with unbounded constraint sets. Optimization 48, 191-210 (2000)

34. Sabharwal, A., Avidor, D., Potter, L.: Sector beam synthesis for cellular systems using phased antenna arrays. IEEE Trans. on Vehicular Tech. 49, 1784$1792(2000)$

35. Sturm, J.F.: Theory and algorithms of semidefinite programming. High performance optimization. Appl. Optim. 33, 1-194 (2000)

36. Todorov, M.I.: Generic existence and uniqueness of the solution set to linear semi-infinite optimization problems. Numer. Funct. Anal. Optim. 8, 541-556 (1985-86)

37. Todorov, M.I.. Uniqueness of the saddle points for most of the Lagrange functions of the linear semi-infinite optimization. Numer. Funct. Anal. Optim. 10, 367-382 (1989) 